\title{
Early Rise in Intraocular Pressure after Ammonia Burns
}

\author{
V. N. HIGHMAN,* M.B., B.S., D.o.
}

Brit. med. F., 1969, 1, 359-360

\begin{abstract}
Cummary : Ammonia is now frequently used in armed robberies by assailants, who possibly misguidedly imagine that it is an easy and safe method of temporarily incapacitating their victim. Nevertheless, two case histories are presented to illustrate the early rise of intraocular pressure which may occur after excessive exposure of the eye to ammonia. The clinical appearance in many respects mimicked that of acute-angle closure glaucoma ; the raised intraocular pressure, corneal oedema, and an oval semi-dilated fixed pupil being typical.
\end{abstract}

\section{Introduction}

In many armed robberies ammonia has been used by the assailant as a method of causing temporary blindness. It has severe toxic effects on the eye which may last some considerable time after the initial insult, and may lead to permanent loss of sight. In two out of seven cases treated for such burns at the Western Ophthalmic Hospital since June 1966 a high intraocular pressure with other signs mimicking acute-angle closure glaucoma were observed at the first examination, which in both cases was within four hours of exposure to ammonia. This association of presenting symptoms in severe ammonia burns to the eye has not so far been recorded, and the following two cases are presented to illustrate it.

\section{Case 1}

During an armed robbery on 20 October 1967 a man aged 57 had ammonia squirted into both eyes. He was seen in the casualty department four hours later with severe burns of the left eye and superficial burns of both lids and lips.

On examination of the left eye the visual acuity was $6 / 18$ unaided. The conjunctiva was grossly injected, chemotic, and necrotic over the tarsus of the lower lid. There was diffuse staining with fluorescein over the entire cornea. The anterior chamber was shallow, the pupil was semi-dilated, oval, and fixed. A red reflex was not obtainable. By applanation tonometry the intraocular pressure was $56 \mathrm{~mm}$. $\mathrm{Hg}$. An accurate assessment of the angle of the anterior chamber was difficult because of the corneal damage, but on gonioscopy it did appear to be open and not unduly narrow.

The visual acuity of the right eye was $6 / 6$ unaided. The conjunctiva was moderately injected and a small central corneal ulcer was noted; the pupil was regular and reacted normally to light. By applanation tonometry the intraocular pressure was $20 \mathrm{~mm}$. $\mathrm{Hg}$ and gonioscopy showed an open angle of medium width.

The patient was admitted, the glaucoma being treated initially with oral glycerol $(1.5 \mathrm{~g} . / \mathrm{kg}$. body weight) and then with oral acetazolamide $250 \mathrm{mg}$. six-hourly and guttae pilocarpin. $4 \%$ halfhourly to the left eye. In order to counter the inflammatory effects of the burn, guttae Predsol (prednisolone sodium phosphate) was instilled four-hourly. On this regimen the intraocular pressure was reduced to $20 \mathrm{~mm}$. $\mathrm{Hg}$ and the conjunctival and corneal ulcers healed. The patient was discharged 18 days after admission on guttae chloramphenicol and prednisolone eight-hourly. Oral acetazolamide $250 \mathrm{mg}$. eight-hourly was continued for a few days after discharge.

Ten days later the left eye became painful and red, the intraocular pressure having risen to $50 \mathrm{~mm}$. $\mathrm{Hg}$. This increase in the intraocular pressure was secondary to closure of the angle of the

* Registrar, Western Ophthalmic Hospital, London N.W.1. anterior chamber by the root of the iris due to the swelling of an intumescent cataract. A large punched-out corneal ulcer was also noted adjacent to the limbus at 9 o'clock. The patient was admitted and the intraocular pressure reduced preoperatively to $20 \mathrm{~mm}$. Hg with acetazolamide $250 \mathrm{mg}$. eight-hourly. An extracapsular cataract extraction was performed the next day, following which the intraocular pressure remained between 15 and $20 \mathrm{~mm}, \mathrm{Hg}$ and the patient was discharged.

One month later the intraocular pressure had again risen, this time to $40 \mathrm{~mm}$. Hg owing to obliteration of the angle of the anterior chamber by peripheral anterior synechiae. The patient was again admitted and the intraocular pressure controlled with oral acetazolamide, following which the peripheral anterior synechiae were divided with a cyclodialysis spatula, a broad iridectomy was performed, and the anterior chamber reformed with air. Surgery was only partially successful, it being necessary to maintain him on acetazolamide $250 \mathrm{mg}$. eight-hourly in order to keep the intraocular pressure below $30 \mathrm{~mm}$. $\mathrm{Hg}$.

After the cataract extraction the visual acuity was $6 / 24$ (+10 D.S. $/+0.5$ D.C. at 180 ), but three months later it had fallen to hand movements owing to progressive corneal oedema. One month later, February 1968, it was learnt that the patient had died, but the cause of death was not discovered.

\section{Case 2}

A man aged 46 was first seen in the casualty department on 10 June 1966 four hours after ammonia had been squirted into his face during a robbery.

On examination he had an uncorrected visual acuity of $6 / 36$ right eye and $6 / 24$ left eye. The lids of the right eye were swollen, the conjunctiva was injected and chemosed, and the cornea was hazy owing to oedema. There was a flare in the anterior chamber but no cells, and the pupil was oval, semi-dilated, and fixed. By applanation tonometry the intraocular pressure was $36 \mathrm{~mm}$. Hg ; gonioscopy was not performed. The left eye was unaffected, the reduced visual acuity being due to refractive amblyopia. By applanation tonometry the intraocular pressure of the left eye was $17 \mathrm{~mm}$. $\mathrm{Hg}$.

$\mathrm{He}$ was initially treated in the casualty department with oral acetazolamide $250 \mathrm{mg}$. six-hourly and guttae prednisolone hourly to the right eye. On acetazolamide the intraocular pressure was reduced to $10 \mathrm{~mm}$. $\mathrm{Hg}$, but admission was necessary four days later owing to an increase in the severity of the anterior uveitis, local steroids having been stopped for two days. He was given a subconjunctival injection of mydricaine and guttae prednisolone hourly to the right eye. On this regimen the anterior uveitis settled and he was discharged after eight days on guttae atropine $1 \%$ daily and guttae Predsol-N six-hourly. He was maintained on this treatment for one month, when a central tarsorrhaphy was done because of recurrent corneal ulceration and diminished corneal sensation. By this time the right eye was quiet and the atropine was stopped, and the local steroids were reduced to twice-daily applications.

Many attempts at tonometry were made, but the results were uncertain because of the difficulty in gaining access to the cornea. In November 1967, one year after the tarsorrhaphy was done, the eye became irritable, there was iris bombé and a dense cataract, and the intraocular pressure was $40 \mathrm{~mm}$. $\mathrm{Hg}$ by applanation, He was admitted and a peripheral iridectomy done, which relieved the iris bombé. Postoperatively the intraocular pressure remained in the region of $30 \mathrm{~mm} . \mathrm{Hg}$ by applanation, but was reduced to and maintained at $12 \mathrm{~mm}$. $\mathrm{Hg}$ when on oral acetazolamide $125 \mathrm{mg}$. sixhourly. Between January and May 1968 the acetazolamide was gradually reduced, so that when last seen, in June 1968, the intraocular pressure was $15 \mathrm{~mm} . \mathrm{Hg}$ by applanation, all treatment having been stopped for six weeks. The cornea, however, was oedematous, 
and there was early vascularization and band-shaped keratopathy. His present visual acuity in the right eye is counting fingers; projection to light is accurate.

It is of interest to note that the visual acuity of the left eye when first seen was $6 / 24$ unaided, the patient stating that this eye had been poor all his life. In August 1966 the refraction for the left eye was +3.50 D.C. at 120 degrees, giving a visual acuity of $6 / 12$. By February 1968 the visual acuity of the left eye had improved to $6 / 5$ with the above correction.

\section{Comment}

Ammonia is a colourless gas with a perceptible odour in a concentration of 50 p.p.m. in air. It is very soluble and combines with water to form ammonium hydroxide. The strongest solution available is between 28 and $29 \%$.

Ocular injury following exposure to ammonia is basically similar to that from other alkalis, with special features attributable to its high solubility in water and lipids and consequent rapid penetration. The effect of this solubility has been investigated by many workers; Siegrist (1920) notably found ammonium ions in the aqueous humour of rabbits' eyes five seconds after the local application of ammonium hydroxide.

Grant (1950) showed that it is the $\mathrm{pH}$ and not the ammonium ion itself that is significant to the degree of injury. Depending on the concentration and duration of contact, all degrees of damage may be seen from mild corneal ulceration to severe damage of the whole anterior segment-loss of corneal epithelium especially in the lower half, stromal oedema, endotheliosis, iritis, lens damage, and corneal anaesthesia with a tendency to sloughing. Grant (1962) mentioned that a rise in the intraocular pressure had been recorded one month after the initial insult.
These two cases illustrate that a rise in the intraocular pressure may occur sooner than we previously thought, since they showed such a rise within four hours of exposure to ammonia.

The other presenting signs in addition to the early rise in the intraocular pressure mimicked in many respects those seen in acute-angle closure glaucoma-namely, the oval semi-dilated non-reacting pupil and corneal oedema. Closure of the angle of the anterior chamber was considered as a likely cause, but in the first case gonioscopy of the affected eye and of its fellow did not confirm this. Gonioscopy in the second case was not performed at the time of the injury, but the anterior chamber of the affected eye was at no time noted to be shallow, and later examination of the unaffected eye showed a medium angle. Considering the speed of penetration of ammonia through the tissues of the eye, inflammatory swelling in the region of the trabecular meshwork could explain this rise in the intraocular pressure. The semi-dilated fixed pupil, however, is more difficult to explain, but considering the speed of penetration of ammonia simultaneous paralysis of both the sphincter and dilator muscles of the iris are distinct possibilities, especially since in both cases there was no return of iris tone. It is interesting to note that Grant (1962) stated that iris damage may not become apparent until the second week after exposure to ammonia.

I wish to thank Mr. Redmond Smith and Mr. R. A. Burn for their kind help and advice, and for allowing me to make use of their cases.

\section{REFERENCES}

Grant, W. M. (1950). Arch. Ophthal., 44, 399

Grant, W. M. (1962). Toxicology of the Eye, p. 35. Springfield, Illinois. Siegrist, A. (1920). Z. Augenheilk., 43, 176.

\section{Medical Memoranda}

\section{Detection by Cytology of Carcinoma-in-situ after Gastroenterostomy}

[With Special Plate facing Page 355]

Brit. med. F., 1969, 1, 360-361

Though gastric cytology has been in use for a long time its value in many situations is only now becoming apparent. It is accepted that the diagnosis of carcinoma of the stomach is more accurate when gastroscopy, radiology, and cytology are used in combination (Blendis et al., 1967). A study of survival rates for the disease shows that in most cases cure by means of surgery would necessitate diagnosis at the carcinoma-in-situ stage. In the intact stomach surface carcinoma diagnosed by cytology has been reported in six cases by Graham (1954), in three cases by Klayman et al. (1955), and in 16 cases by Schade (1960). The diagnosis of carcinoma after partial gastrectomy or gastroenterostomy is more difficult, but cytology has been used in three cases after partial gastrectomy (Gibbs, 1962); all were found to be extensive at operation. Passarelli et al. (1963) reported a carcinoma-in-situ which developed in the gastric remnant three years after partial gastrectomy and gastroenterostomy for an adenocarcinoma of the greater curvature. Here we report the diagnosis of carcinoma-in-situ by cytology 23 years after gastroenterostomy.

\section{CASE Report}

The patient, a man aged 48, developed symptoms of duodenal ulceration in 1940. Haematemesis occurred in 1941, 1942, and
1943. The ulcer then perforated and was treated by simple closure. In 1944 pyloric stenosis developed, and a retrocolic gastroenterostomy was performed. Upper abdominal pain related to meals continued, and there were several further haematemeses, before vagotomy was performed in 1963 . Gastric secretory studies were not performed. At the age of 47 he was admitted to the Edinburgh Royal Infirmary with complaints of tiredness and nausea. He was anaemic, with haemoglobin 9.8 g. $/ 100 \mathrm{ml}$., M.C.H.C. $29 \%$, serum iron $29 \mu \mathrm{g} . / 100 \mathrm{ml}$., and total iron-binding capacity $570 \mu \mathrm{g} . / 100 \mathrm{ml}$. Stool occult blood was negative. A barium-meal examination showed minor irregularity of the greater curvature of the antrum. The patient was achlorhydric to pentagastrin (Peptavlon $6 \mu \mathrm{g}$./ kg.), the minimum $p \mathrm{H}$ being $7 \cdot 3$. Intrinsic factor output was $1,056 \mathrm{~m} \mu \mathrm{g}$. in the post-pentagastrin hour. Tests for parietal cell and intrinsic factor antibodies were negative. Gastric lavage carried out with two $300-\mathrm{ml}$. aliquots of normal saline gave recoveries of 160 and $120 \mathrm{ml}$. Malignant cells were found (Fig. 1). At laparotomy no abnormality of the stomach was seen, but a Billroth I antrectomy was carried out. A further gastric lavage performed six months after operation showed active epithelial cells but no malignant cells.

\section{Pathology}

Exfoliative cytology revealed clumps of cells which were clearly different from the palely staining gastric epithelial cells (Fig. 1). The former showed hyperchromatic nuclei with large and prominent nucleoli and darkly staining basophilic cytoplasm.

The lesion itself consisted of a velvety patch of rather irregular but moderately well circumscribed mucosa measuring 2.5 to $1.5 \mathrm{~cm}$. 